\title{
A commentary on fine mapping and resequencing of the PARK16 locus in Parkinson's disease
}

\author{
Joanne Trinh, Carles Vilariño-Güell and Owen A Ross \\ Journal of Human Genetics (2015) 60, 405-406; doi:10.1038/jhg.2015.76; published online 2 July 2015
}

$\mathrm{I}$

t has been almost two decades since the first genetic form of Parkinson's disease (PD), a point mutation in the $\alpha$-synuclein gene (SNCA), was identified. ${ }^{1}$ Remarkable technological advancements in recent times have accelerated genetic discovery for PD and nominated potential molecular pathways for therapeutic intervention strategies. To date, pathogenic mutations in six genes (SNCA, LRRK2, VPS35, PARKIN, PINK1 and DJ-1) have been confirmed to result in carriers presenting the clinical phenotype associated with $\mathrm{PD}$, with the vast majority of these reporting a family history of the disease. ${ }^{1}$ Furthermore, a recent large-scale metaanalysis of population-based genome-wide association studies (GWAS) has identified 24 independent genomic loci (including the familial genes SNCA and LRRK2) that influence susceptibility to the common sporadic form of PD. ${ }^{2}$

The loci nominated through GWAS approaches tend to be large genomic regions defined by patterns of linkage disequilibrium (LD) and contain multiple candidate genes. Thus, identifying the variant (or gene) responsible for modifying disease susceptibility within these loci remains challenging. In the previous issue, Pihlstrøm et al. ${ }^{3}$ attempt to fine-map and resequence the PARK16 locus in patient populations from Scandinavia. PARK16 was one of the first loci nominated through GWAS efforts in both Asian and European cohorts. ${ }^{4,5}$ Although replications in Asian populations were consistently supportive, studies in Caucasian populations were initially controversial. ${ }^{3}$ The

J Trinh and C Vilariño-Güell are at Department of Medical Genetics, University of British Columbia, Vancouver, British Columbia, Canada; OA Ross is at Department of Neuroscience, Mayo Clinic, Jacksonville, FL, USA

E-mail: carles@can.ubc.ca or ross.owen@mayo.edu recent large-scale meta-GWAS in Caucasian samples has replicated the association and would appear to confirm this disease locus across ethnicities. ${ }^{2}$ From these studies it would appear that PARK16 contains five candidate genes (SLC45A3, NUCKS1, RAB7L1, SLC41A1 and PM20D1) and it remains unclear which specific gene and/or variant(s) is responsible for the observed association.

The authors had previously attempted to replicate the association at the PARK16 locus using three single-nucleotide variants (rs947211, rs823128 and rs823156) in these populations and no association was observed. ${ }^{6}$ However, in the present study the authors report a different haplotype spanning the transcription start site for RAB7L1 (rs974211-rs1572931-rs1775143) associating with disease risk $(P=0.046)$. Pihlstrom and colleagues ${ }^{7}$ also replicated a recently nominated epistatic interaction between this haplotype and a LRRK2 GWAS association with the variant rs1491942. Targeted resequencing of the coding regions of the candidate genes identified a low-frequency risk variant in PM20D1 (rs141605758); however, after further genotyping in a larger case-control cohort, there was no convincing evidence of pathogenicity. From these data the authors conclude that RAB7L1 is the most compelling candidate in the PARK16 locus in the Scandinavian population.

The thrust of the study and the level of allelic heterogeneity observed in replication attempts suggest the existence of different functional variants or one variant of independent origin within the PARK16 locus. The most recent large-scale meta-GWAS has indicated an association at the $3^{\prime}$ untranslated region of $R A B 7 L 1,{ }^{2}$ whereas the strongest association in the initial Caucasian GWAS study was in NUCKS1 (rs823121), upstream of $R A B 7 L 1 .^{5}$ Similarly, the strongest association in the Japanese population also lies upstream of RAB7L1 (rs947211), ${ }^{4}$ whereas in the Chinese population both downstream (rs823156) and upstream (rs823128) polymorphisms of RAB7L1 were most significantly associated with PD risk. ${ }^{8}$ These differences suggest that elucidating the functional variant for PARK16 may be particularly challenging as the associations appear dependent on population-specific LD structure and thus different populations may harbor separate risk factors. This scenario means that targeted resequencing of the entire genomic locus in homogenous populations such as described may prove fruitful in resolving the functional variant driving risk.

Differences in population structure are highlighted by Pihlstrom et al. within the Scandinavian population as the risk alleles in RAB7L1 (rs1775143) had a larger effect size in Sweden (odds ratio (OR) 0.76) compared with Norway (OR 0.91). Perhaps, conditional analysis with multiple common variants across PARK16 will help reveal independent association signals for each population. In addition to the genetic evidence, $R A B 7 L 1$ is a very attractive biological candidate; the protein is part of the endosomal-lysosomal pathway and has been shown to interact with both LRRK2 and VPS35. ${ }^{7,9}$ RAB7L1 localizes to the trans-Golgi network, and retromer/vesicular trafficking has become an emerging pathway since the implication of VPS35 and DNAJC13 in PD. ${ }^{10,11}$

Although the causal variant in PARK16 is yet to be identified, it does not seem to be a simple amino-acid change: no mutations in the coding regions of the candidate genes that could account for the observed association were observed by Pihlstrom and colleagues. This suggests that the mutation is likely 
within a noncoding region of the locus and would support the application of nextgeneration sequencing approaches to tease out the functional variant(s). Noncoding variation can affect splice sites, transcription factor-binding sites or methylation sites, modulating splicing and gene expression, which is difficult to identify without appropriate tissue types and functional readouts. Identifying the truly functional variants at this locus and others may prove difficult. Nonetheless, at PARK16, the genetic association of $R A B 7 L 1$ and the biological relevance of the protein in the pathogenesis of $\mathrm{PD}$ are becoming more convincing and further investigation to define the pathogenic mechanism is warranted.

\section{CONFLICT OF INTEREST}

The authors declare no conflict of interest.
1 Trinh, J. \& Farrer, M. Advances in the genetics of Parkinson disease. Nat. Rev. Neurol. 9, 445-454 (2013).

2 Nalls, M. A., Pankratz, N., Lill, C. M., Do, C. B., Hernandez, D. G., Saad, M. et al. Large-scale meta-analysis of genome-wide association data identifies six new risk loci for Parkinson's disease. Nat. Genet. 46, 989-993 (2014).

3 Pihlstrøm, L., Rengmark, A., Bjørnarå, K. A., Dizdar, N., Fardell, C., Forsgren, L. et al. Fine mapping and resequencing of the PARK16 locus in Parkinson's disease. J. Hum. Genet. 60 357-362 (2015).

4 Satake, W., Nakabayashi, Y., Mizuta, I., Hirota, Y. Ito, C., Kubo, M. et al. Genome-wide association study identifies common variants at four loci as genetic risk factors for Parkinson's disease. Nat. Genet. 41, 1303-1307 (2009).

5 Simon-Sanchez, J., Schulte, C., Bras, J. M. Sharma, M., Gibbs, J. R., Berg, D. et al. Genome-wide association study reveals genetic risk underlying Parkinson's disease. Nat. Genet. 41 1308-1312 (2009).

6 Pihlstrom, L., Axelsson, G., Bjornara, K. A., Dizdar, N., Fardell, C., Forsgren, L. et al. Supportive evidence for
11 loci from genome-wide association studies in Parkinson's disease. Neurobiol. Aging 34 1708e7-13 (2013).

7 MacLeod, D. A., Rhinn, H., Kuwahara, T., Zolin, A. Di Paolo, G., McCabe, B. D. et al. RAB7L1 interacts with LRRK2 to modify intraneuronal protein sorting and Parkinson's disease risk. Neuron 77, 425-439 (2013).

8 Tan, E. K., Kwok, H. H., Tan, L. C., Zhao, W. T. Prakash, K. M., Au, W. L. et al. Analysis of GWAS-linked loci in Parkinson disease reaffirms PARK16 as a susceptibility locus. Neurology $\mathbf{7 5}$, 508-512 (2010).

9 Beilina, A., Rudenko, I. N., Kaganovich, A., Civiero, L. Chau, H., Kalia, S. K. et al. Unbiased screen for interactors of leucine-rich repeat kinase 2 supports a common pathway for sporadic and familial Parkinson disease. Proc. Natl Acad. Sci. USA 111, 2626-2631 (2014).

10 Vilarino-Guell, C., Rajput, A., Milnerwood, A. J., Shah, B., Szu-Tu, C., Trinh, J. et al. DNAJC13 mutations in Parkinson disease. Hum. Mol. Genet. 23 1794-1801 (2014).

11 Vilarino-Guell, C., Wider, C., Ross, O. A., Dachsel, J. C. Kachergus, J. M., Lincoln, S. J. et al. VPS35 mutations in Parkinson disease. Am. J. Hum. Genet. 89 162-167 (2011). 